\title{
GOBERNANZA Y RÉGIMEN URBANO: MÁS ALLÁ DE LA VISIÓN DEL INTERRUPTOR
}

\author{
GOVERNANCE AND URBAN REGIME: BEYOND THE LIGHT SWITCH APPROACH
}

Francisco Javier Porras Sánchez*

\section{RESUMEN}

En los últimos años, las literaturas de la gobernanza y las Teorías del Régimen Urbano (TRU) se han consolidado como referentes importantes en la renovación del interés por lo local. Las dos hacen aportes desde distintos presupuestos teóricos y empíricos y, aunque con diferencias sustantivas, comparten algunos problemas fundamentales. El artículo argumenta que la consideración de algunos de ellos (el «motor explicativo», las élites económicas locales y las coaliciones, la agenda urbana irresistible o convincente y el problema de la medición, entre otros) confirma la conveniencia de usar un enfoque analítico en lugar de uno meramente normativo. Esta aproximación, particularmente evidente en los últimos desarrollos de la gobernanza, sería útil para impulsar una agenda de investigación de las TRU que fuera más allá de la preocupación usual por comprobar la existencia de tales regímenes en contextos diferentes al norteamericano.

Palabras clave: Teorías del Régimen Urbano, teorías de la gobernanza, agenda urbana, élites económicas, medición.

\section{ABSTRACT}

In recent years, the academic literatures of governance and the Theories of Urban Regime (TUR) have consolidated into important references in the interest renewal for the local. Both make proposals with distinct theoretical and empirical assumptions and, although with substantial differences, they share some fundamental problems (the "explanatory motor», the local economic elites and coalitions, the irresistible or convincing urban agenda, and the problem of measuring, among others). The article argues that their consideration confirms the convenience of using an analytical approach rather than one that is merely normative. This focus, particularly evident in recent developments of governance literature, is useful for furthering a TUR research agenda beyond the usual concern for proving the regimes' existence in contexts other than American ones.

Keywords: Theories of Urban Regimes, governance theories, urban agenda, economic elites, measuring.

Recibido II de diciembre de 2020 y aceptado 30 de abril de 2021

\footnotetext{
${ }^{*}$ Profesor-Investigador del Instituto de Investigaciones Dr. José Ma. Luis Mora
} (Instituto Mora).<fporras@institutomora.edu.mx>. 


\section{LA APROXIMACIÓN DEL INTERRUPTOR DE LUZ}

$\mathrm{Al}$ igual que las literaturas académicas de la gobernanza local y la gobernanza urbana, la teoría del régimen Urbano (TRU) ha desempeñado un papel central en lo que John (200I: 3) llama «la reinvención de la política local». La perspectiva del régimen urbano introdujo elementos conceptuales que no solamente visibilizan las interdependencias que existen entre actores gubernamentales y no-gubernamentales para gobernar una ciudad, sino también establece correlaciones entre recursos y decisiones, entre estructura y agencia, que explican la formación y el mantenimiento de las agendas urbanas estratégicas, y cómo estas son impulsadas a través de coaliciones más o menos duraderas. La TRU es un referente indispensable para entender las diferentes formas que adquiere el poder y cómo este impulsa, limita y da forma al crecimiento físico y económico de la ciudad (Vélez Dávila, 2013).

Sin embargo, de la misma manera que las literaturas mencionadas, la TRU enfrenta el reto de consolidar sus propuestas teóricas, empíricas y metodológicas a través de los estudios comparados. Esto requiere más que simplemente definir qué es un régimen urbano, incluyendo sus variantes o tipologías, y establecer si el modelo puede «viajar» bien en el tiempo y en el espacio -lo que muchas veces ha reducido la sofisticada discusión en torno a la TRU a únicamente explorar si existen regímenes urbanos fuera de las ciudades norteamericanas (Pierre 20I4) y qué implicaciones tiene esto para hipotetizar un(os) posible(s) «proyecto(s) urbano(s) hegemónico(s)» (Ciccolella y Mignaqui 20I6: I73). Para consolidar las propuestas de la TRU se necesitan identificar claramente los problemas centrales que intenta explicar, independientemente de los cambiantes contextos espacio-temporales, reconociendo su utilidad analítica para ayudar a gestionar la complejidad de las ciudades.

En efecto, las literaturas de la gobernanza local y la gobernanza urbana en particular, pero también la TRU en cierto grado, frecuentemente han sido tratadas como modelos más o menos acabados que solamente necesitan ser llevados a campo para verificar o no su cumplimiento. Esta perspectiva, usual en investigaciones de posgrado, busca comprobar -o en su caso desecharque las respectivas definiciones conceptuales se cumplen en los contextos específicos, para luego aplicar las correlaciones establecidas en la teoría. Así, 
se pone énfasis en demostrar que existe o no la gobernanza o el régimen urbano, para después explicar los hallazgos desde una perspectiva que se diferencia poco de los enfoques normativos tradicionales.

$\mathrm{El}$ argumento de fondo pareciera proponer algo similar a esto: «(a) Un régimen urbano se caracteriza por $\mathrm{x}, \mathrm{w}$ y z; (b) en esta ciudad se puede verificar $\mathrm{x}, \mathrm{w}$ y z; en consecuencia, (c) en esta ciudad existe un régimen urbano». De esa primera conclusión se seguiría que a la ciudad estudiada se le pueden aplicar de manera cuasi-automática los presupuestos teóricos, empíricos y metodológicos que acompańan a la TRU; por ejemplo, explicar siempre la conducción del desarrollo urbano desde una coalición que es capaz de subordinar a otras redes público-privadas, o asignar inmediatamente un valor central a los(as) actores(as) locales, por encima de otros(as). De la misma manera, si el trabajo de campo verifica que las condiciones se cumplen solamente de manera parcial, se abre la discusión de si es posible aplicar la teoría al caso y de qué modo. Esto ha llegado a límites tales como preguntarse si hay que operacionalizar las variables de las definiciones, medirlas a través de indicadores y luego establecer una línea -por ejemplo, un porcentaje- que permita distinguir entre casos (véase por ejemplo a Rhodes, 2000).

A la larga, esta aproximación de «interruptor de luz» («sí existe»/«no existe»; "prendido»/«apagado») no es sustentable, pues lo usual es que la realidad social contradiga la teoría de muchas maneras y en distintos niveles, generando estadios intermedios entre el «on» y el «off». De igual manera, la teoría puede hacer aportes sustantivos para el entendimiento de los contextos, independientemente de que se cumplan todos los prerrequisitos establecidos. Las literaturas de la gobernanza y la TRU no aspiran a explicar todas las dimensiones de la conducción del desarrollo urbano, en todos los entornos. Sin embargo, esto no demerita su valor.

Aguilar (2006) ha argumentado que es indispensable ir más allá de los enfoques descriptivos, normativos y prescriptivos, que pueden violentar la realidad o ser decepcionados por ella, para construir aproximaciones que pongan énfasis en el poder analítico de las teorías. Siguiendo esta invitación y a manera de ejemplo, Cadena Inostroza y Salgado Locela (2017: 62) encuentran una cierta utilidad en las propuestas de la gobernanza local para el análisis de un municipio del Estado de México, siempre y cuando no se considere la teoría como un modelo cerrado y terminado, sino como una 
oportunidad para distinguir y separar los distintos actores, instituciones y recursos involucrados en los procesos para acordar y ejecutar objetivos comunitarios. Para estas autoras, la gobernanza local debe entenderse como la «diversidad de configuraciones que surgen en espacios de conflicto de intereses», lo que no solo permite considerar una gran variedad de configuraciones públicas-sociales-privadas para el logro de objetivos comunes, sino que -crucialmente- deja en un segundo plano el problema de si existe o no la gobernanza local en determinado lugar. Desde esta perspectiva, las teorías de la gobernanza son instrumentos para plantear y resolver problemas sobre el timoneo en contextos de mayor fragmentación sociopolítica o en los que los actores no-gubernamentales son más numerosos o importantes (Porras, 2019).

Una aproximación analítica, que considere los problemas fundamentales implícitos en las teorías, tiene dos ventajas de peso. La primera es que permite ir más allá del problema del contexto que originó las categorías y conceptos, recolocando la discusión en las variables, correlaciones o relaciones causales que intentan entender/explicar procesos básicos comunes a diferentes entornos. En este sentido, los problemas fundamentales se identifican con categorías, rangos (continuos) y tensiones que han sido frecuentemente tratados, de una manera u otra, por las ciencias sociales. En consecuencia, y esta es la segunda ventaja, las teorías pueden mostrar su utilidad-siempre parcial- en un lenguaje y usando un enfoque apropiado al diálogo interdisciplinario. Los problemas fundamentales usualmente son objetos de estudio comunes a varias disciplinas.

Este artículo argumenta que así como en los casos de las literaturas de la gobernanza local y la gobernanza urbana, la TRU se beneficiaría de un tratamiento que ponga énfasis en sus problemas fundamentales, yendo más allá de la cuestión de si pueden existir o no regímenes urbanos fuera de Atlanta, Georgia. Más específicamente, la TRU parece hacer aportes sustantivos para el entendimiento de la reproducción de los sistemas sociales que permiten gobernar una ciudad, y la interdependencia que existe entre la agenda urbana estratégica y los recursos necesarios para llevarla a cabo (esta última conocida como la «ley de hierro» de los regímenes urbanos [Stone 2002: IO]). Estos problemas constituyen su motor explicativo, funcionando como un diferenciador importante respecto a las literaturas de la gobernanza. 
El artículo explica este argumento a través de tres secciones principales. En la primera se analizan brevemente los principales aportes de las TRU, identificando sus atributos principales de acuerdo con algunos autores canónicos. La segunda parte elabora sobre algunos problemas fundamentales de las TRU, particularmente los que comparte con las teorías de la gobernanza (la interdependencia como motor explicativo, élites y coaliciones, agenda urbana «irresistible»/convincente, y los problemas de la medición). Finalmente, en la última sección se presentan las conclusiones generales del artículo.

\section{LAS TRU}

Como nos recuerdan Judge, Stoker y Wolman (1998: 2-3), las teorías en las ciencias sociales usualmente adquieren la forma de teorías normativas/prescriptivas - las cuales buscan «establecer los mejores medios para lograr los fines deseados»-; de teorías empíricas -que tratan de "explicar e interpretar la realidad»-; de modelos -que son representaciones estilizadas y simplificadas de la realidad-, y de marcos conceptuales o perspectivas -que son «maneras de ver o concebir un objeto de estudio». Desde esta clasificación mínima, uno puede identificar distintos presupuestos conceptuales, niveles de análisis, alcances explicativos y contextos originarios.

En el caso de las TRU, dependiendo del autor de que se trate y a primera vista, pareciera que es usual interpretar las categorías conceptuales propuestas originalmente por Stone (1989) como teorías empíricas, como modelos o como marcos conceptuales/perspectivas. Las teorías empíricas y los modelos tienden a ser más específicos, definiendo al régimen urbano de manera más estrecha -i.e. enumerando una mayor cantidad y especificidad de atributos-, mientras que los marcos conceptuales/perspectivas son más amplios -y en ese sentido quizá tienen mayor utilidad para desarrollar enfoques analíticos.

Usando una aproximación estrecha, un régimen urbano se puede definir como el conjunto de «arreglos informales que rodean y complementan los trabajos formales de la autoridad gubernamental» (Stone, 1989: 3). Son

[...] los arreglos informales a través de los cuales instituciones públicas e intereses privados funcionan juntos para realizar y llevar a cabo deci- 
siones de gobierno. Estas decisiones de gobierno [...] no tienen que ver con hacer o controlar todo. Tienen que ver con la administración del conflicto y dar respuestas adaptadas al conflicto social (Stone, 1989: 6).

Un régimen urbano es el conjunto de arreglos informales, pero relativamente estables, a través de los cuales se gobierna una localidad. Por gobernar quiero decir obtener los recursos necesarios para buscar implementar [...] una dirección de política pública estratégica [...]. Gobernar no es el control comprehensivo de todas las decisiones, muchas de las cuales ocurren a través del procesamiento paralelo de subsistemas [...] Gobernar implica el establecimiento de una agenda que define una dirección. Para definir una dirección, una agenda debe tener, en lo principal, una prioridad para toda la localidad. Entre más fuerte el régimen, más firme la prioridad. Si la prioridad de la agenda es débil, el régimen es débil (Stone, 2002: 7).

Un régimen urbano implica (i.e. requiere):

a) Un grupo informal de actores. que, sin embargo, es estable

b) Con acceso a recursos institucionales

c) Que buscan el cumplimiento de un objetivo (agenda estratégica)

d) Que mantienen un papel sostenido en las decisiones de gobierno, y

e) Que no buscan ni son capaces de controlar todo, sino que gestionan el conflicto de la ciudad (Stone, 1989: 4-6) (cursivas mías)

De manera más específica, y desarrollando las implicaciones de estos atributos, Stoker (1998) argumenta detalladamente que las TRU

a) Reconoce la interdependencia entre actores gubernamentales y no-gubernamentales para atender problemas sociales y económicos. Las TRU parten del supuesto que algún tipo de cooperación existe entre actores públicos y privados.

b) Tiene en el centro el problema de la gobernanza (i.e. considera a los gobiernos locales trabajando a la par de otros intereses, en un contexto social, político y/o económico complejo).

c) Redirecciona la comprensión del poder del control social (e.g. a través de jerarquías o gobernabilidad) a un proceso de producción social. 
Esto requiere visualizar el problema de la conducción del desarrollo de una ciudad como el producto de condiciones estructurales -que se expresan en formas diversas.

d) Presupone una serie de instituciones controladas por el voto popular (democracia representativa), combinadas con una economía impulsada fundamentalmente por inversiones privadas.

e) Su premisa fundacional es que los «tomadores de decisiones urbanos tienen una autonomía relativa»; el poder sistémico (i.e. estructural) pone límites a la acción de los actores; pero la política es todavía posible dentro de estos límites (Stoker, I998: 56). Dicho de otra manera, aunque Stone (1989) reconoce la importancia de la estructura sobre la agencia, de ninguna manera propone dinámicas deterministas de una sobre otra.

f) Reconoce contextos urbanos de mayor complejidad, lo que no facilita el control de procesos políticos y económicos por un solo actor. El gobierno consolida su lugar central como coordinador y movilizador de recursos. Las TRU parten de un escenario similar al que proponen las literaturas de la gobernanza.

g) Para lograr sus objetivos, los gobiernos deben mezclar (blend) sus capacidades con las de los actores privados; los actores privados forman regímenes con los públicos, lo que facilita la toma de decisiones y el empoderamiento de los actores no-gubernamentales.

h) Las TRU son, en última instancia, «un modelo de elección pública en el entorno urbano» al se le da forma a través de una coalición de gobierno, la naturaleza de la relación de los miembros de tal coalición, y los recursos que los miembros poseen y traen a la coalición (Stoker, I998: 6I).

Las TRU parten del supuesto de que los regímenes urbanos no explican toda la complejidad de las redes público-sociales-privadas que posibilitan el gobierno de una ciudad, pero sí aspiran a identificar las coaliciones y recursos que definen una agenda como prioritaria (estratégica) tanto para los miembros de la coalición del régimen, como para otros actores e instituciones. Sin embargo, esto no implica la subordinación automática y jerárquica de otras redes público-sociales-privadas a la coalición hegemónica. 
Las TRU no proponen una aproximación de redes de política pública (o redes de «gobernanza neoliberal», como las llama Palumbo (20I5: 73) formadas exclusivamente por élites políticas y económicas poderosas. Un «régimen urbano no es sinónimo de una élite encubierta y cerrada» (Stone, 2002: 7) porque, entre otras razones, tanto la agenda estratégica como los recursos movilizados para cumplirla están ampliamente distribuidos. Aún más interesante, Stone (1998) argumenta que la estructura que mantiene el régimen no es una basada solamente en procesos de política pública -comandados por los funcionarios locales- interactuando con los intereses capitalistas de los negocios locales -liderados por los hombres y mujeres de negocios-, sino que se mantiene en última instancia por procesos de reproducción social en los que imágenes, narrativas y valores se usan para establecer que un determinado tema urbano tiene precedente sobre otros. Así, usando la analogía de la ley de gravedad, Stone (1989: 235) propone que entre más actores reproducen el régimen, este se mantiene más fácilmente.

Considerando al régimen urbano desde una aproximación más amplia -la del marco conceptual/perspectiva-, los atributos mencionados tienden a ser menos numerosos, poniendo énfasis en criterios heurísticos -i.e. de interpretación- que permiten visibilizar las variables centrales de la teoría. Así, por ejemplo, Mossberger y Stoker (200I: 829) argumentan que el enfoque de las TRU nos permiten identificar (a) los procesos de colaboración basados en la producción social (dada la necesidad de combinar recursos para lograr objetivos); (b) las coaliciones entre socios públicos y privados, requiriendo la participación de los negocios de la ciudad -aunque de manera no exclusiva-; (c) las agendas vinculadas a los integrantes de las coaliciones, y (d) los patrones de colaboración público-privados que se mantienen en el tiempo. Vélez Dávila (2013: 66-67) propone, de una manera más sencilla, que los regímenes urbanos resultan de la existencia de (I) una agenda urbana, (2) una coalición para lograrla, (3) esquemas varios de colaboración, y (4) la movilización de recursos público-privados.

Las TRU ayudan a evidenciar que el desarrollo de algunas ciudades es el resultado de (a) una agenda compartida entre actores públicos y privados, (b) las coaliciones que buscan lograr tal agenda a través de diversos mecanismos de colaboración, y (c) la movilización de recursos de -principalmente- el gobierno y los negocios para cumplirla. De esta manera, el régimen urbano 
es una categoría que podría ser considerada como posburocrática o posgubernamental, en el sentido de que subraya la colaboración entre diversos tipos de actores para el logro de objetivos de la ciudad, sin considerar al gobierno formal necesariamente como el único o más relevante actor.

\section{ALGUNOS PROBLEMAS FUNDAMENTALES}

Al revisar las diferentes definiciones y categorías aportadas por las TRU, resaltan algunos temas similares a los tratados por otras literaturas académicas, más notablemente las de la gobernanza local y de la gobernanza urbana, pero en los cuales se diferencia de manera importante. Aquí se analizan brevemente cuatro, conforme se explica a continuación.

\section{GOBERNANZA Y MOTOR EXPLICATIVO}

El primero de ellos es el problema de la gobernanza y el «motor explicativo» del régimen urbano. Es interesante que tanto la literatura de la gobernanza social (societal governance) de Kooiman (2003) como las TRU presuponen que las sociedades actuales son más difíciles de dirigir, ya que los actores no-gubernamentales no solamente se encuentran más especializados respecto a sus intereses y sus capacidades para agregarlos, sino que poseen más información, legitimidad, dinero, expertise y conocimiento técnico en sus respectivos ámbitos de influencia. Sin embargo, estos recursos que se encuentran más dispersos son necesarios para resolver los problemas complejos de la ciudad -o para dirigirla hacia el cumplimiento de algunos objetivos que se consideran centrales-, por lo que algún tipo de cooperación -generalmente informal- es indispensable. Esta tensión entre la dispersión de los recursos, por un lado, y la necesidad de cooperación, por el otro, es central para explicar el régimen urbano. Como argumenta Stone (1989: 219),

[...] las sociedades modernas se caracterizan por tener fuertes fuerzas centrífugas. Fragmentadas en miríadas de roles especiales, estas sociedades carecen de un poder de mando que abarque todo. La autoridad formal del gobierno es limitada y una cantidad substantiva de recursos 
está en manos privadas. Especialmente en el nivel local, el poder de los funcionarios públicos puede ser reducido por procesos y actividades fuera del control gubernamental. Dado que las comunidades están divididas en varios sectores con muchos actores independientes en cada uno de ellos, la cooperación a través de las líneas institucionales no se puede lograr simplemente por acciones formales. Así, la cooperación lograda informalmente puede ampliar la efectividad del régimen.

El conflicto entre las «fuerzas centrífugas» y la necesidad de actuar en conjunto da forma a las diferentes modalidades de gobernanza de la ciudad. En este punto, y a primera vista, la gobernanza y el régimen urbano parecen indistinguibles, con sutiles diferencias de enfoque más que desacuerdos sustanciales. La gobernanza pone atención en los procesos de acuerdo e implementación de objetivos comunes -i.e. el direccionamiento-; mientras que el régimen urbano enfatiza el gobierno de la ciudad a través de alianzas, tradicionalmente con actores del mundo de los negocios. La gobernanza se puede mantener con actores de los mercados, aunque usualmente considera un rango en el que los actores e instituciones sociales juegan un papel importante. Las TRU, por otro lado, no excluyen a actores sociales, pero los consideran desde el punto de vista de los procesos de reproducción social.

En términos generales, la gobernanza puede verse como un conjunto de problemas teóricos, empíricos y metodológicos que resultan de los procesos de codirección en entornos de mayor fragmentación sociopolítica (Porras, 20I9). También puede verse como un «patrón flexible de decisiones públicas basado en redes sueltas (loose) de individuos» (John, 200I: 9). La gobernanza, particularmente de las ciudades, implica procesos complejos entre los que se encuentran (a) la reforma institucional (la multiplicación y la restructuración institucionales); (b) la presencia de nuevas redes (redes horizontales más fuertes y nuevas redes trasnacionales); (c) las nuevas iniciativas de política pública (de innovación local y construcción de capacidad, así como nuevas iniciativas del gobierno nacional), y (d) las respuestas a dilemas de coordinación y rendición de cuentas (la búsqueda de nuevos mecanismos de control y rendición de cuentas, así como las formas más prominentes de liderazgo ejecutivo) (John, 200I: I5-I6). 
En el centro de sus preocupaciones se encuentran (I) el timoneo (codirección) para el cumplimiento de objetivos comunes; (2) la coordinación/cooperación entre distintos actores para cumplir tales objetivos; (3) los procesos de control y comunicación -a veces considerablemente más «suaves» que en el pasado-, y (4) la rendición de cuentas, tanto de funcionarios públicos como de actores e instituciones no-gubernamentales (Aguilar, 2019). Al igual que las TRU, las literaturas de la gobernanza identifican muchos de estos problemas como centrales a sus respectivos intereses y agendas de investigación (Guarneros-Meza, 2006: 67), al grado de que algunos autores consideran a los regímenes urbanos son un subtipo de redes de política pública (Guarneros-Meza, 2006), o como el "pináculo del proceso de gobernanza» en el que la «formación de redes, la creación de confianza y la solución de problemas» es fundamental (John, 200I: 52).

Algunas literaturas académicas han identificado procesos similares a los analizados por las TRU caracterizándolos como ejemplos de «gobernanza emprendedora»-i.e. «intentos del sector público por garantizar e incentivar la inversión a través de programas de reducción de impuestos y la promoción de ciudades como si fueran mercancías»- (McCann, 20I7: 314). Palumbo (20I5: 73) habla de la «gobernanza neoliberal»-como se mencionó arribaargumentando que, en muchas ciudades, la inversión para el crecimiento se deja en manos del mercado y que los servicios públicos son frecuentemente prestados bajo esquemas de alianzas público-privadas (Public-Private Partnerships, o PPP). Davies (2OII) argumenta que mucho de la narrativa de la gobernanza es resultado de la hegemonía del modelo neoliberal, que ha tenido como resultado la privatización de la política en general y de los procesos de política pública local en particular (Guarneros-Meza, 2007, 2008, 20II).

Pierre (20II), por otro lado, llama «gobernanza pro-crecimiento» a las coaliciones público-privadas que buscan el desarrollo económico de las ciudades, mientras que -de manera más amplia y con una perspectiva más sociológica- Emerson y Nabatchi (2015) proponen la categoría de «regímenes colaborativos», y Gash (20I6) la de "gobernanza colaborativa». Finalmente, Ansell y Torfing (2016: 559) reconocen que el estudio detallado de los procesos multi-actor, entre los que se encuentran los regímenes urbanos, debería ser parte de la agenda de investigación de las literaturas de la gobernanza. Estos autores llaman la atención particularmente a la combinación 
de modelos de interacción público-privados y a las dinámicas de liderazgo gubernamental y no-gubernamental.

A pesar de compartir algunos problemas de investigación, presupuestos teóricos, hallazgos empíricos y herramientas metodológicas, las TRU tienen al menos dos diferencias sustantivas con los enfoques de la gobernanza. La primera es que la gobernanza tiende a subrayar los procesos de codireccionamiento (i.e. las diferentes fases y componentes institucionales que permiten acordar objetivos público-privados y llevarlos a cabo) usualmente definidos en términos de Nuevos Instrumentos de Política Pública (NIPps) -i.e. herramientas de timoneo que usan la información y el convencimiento voluntario en lugar de la coerción legal. Las TRU, por otro lado, tienden a ser considerablemente más amplia y -si se me permite la expresión- profunda, en el sentido de que pone la mirada en procesos de reproducción social que dan por resultado agendas urbanas estratégicas. Es cierto que estas agendas son mantenidas e impulsadas activamente por redes de miembros de la coalición informal del régimen; sin embargo, las TRU conciben los temas prioritarios como fruto de intereses específicos en contextos de cultura política, mientras que a la gobernanza le preocupan los problemas más inmediatos de cómo, a través del consenso y el uso de NIPPs, se forman redes público-sociales-privadas.

En otras palabras, y quizá con riesgo de simplificar demasiado, a la gobernanza le interesan las redes de cogobierno (su estructura, funcionamiento y gestión), mientras que las TRU estudian tales redes en el contexto estructural que permite su formación, actividad, permanencia y modificación. La idea de que las redes no existen en el vacío y deben ser analizadas en su contexto es un desarrollo relativamente nuevo en las literaturas de la gobernanza. De hecho, fue hasta la entrada de la literatura del anclaje democrático en el mainstream de la gobernanza con Skelcher, Klijn, Kübler, Sørensen y Sullivan (20II) que se empezó a explicar qué sucedía dentro de las redes usando variables estructurales (el milieu democrático y el capital social, en este caso). La relevancia de la estructura, sin embargo, ya había sido propuesta por las TRU al argumentar un «modelo de producción social» más que «de control social» (Davies y Blanco, 20I7: 3).

Otra diferencia sustantiva es el motor explicativo de las teorías. Para la gobernanza, la paradoja de tener «mandatos incumplibles» en los gobier- 
nos (i.e. que a pesar de tener el mandato legal y una alta concentración de recursos informacionales, financieros, tecnológicos, de legitimidad y de expertise pueden encontrarse en la situación de no poseer todos los recursos necesarios para cumplir sus objetivos) ha fomentado su fragmentación en diversos sectores de política pública, y la apertura de sus redes a actores no-gubernamentales (Rhodes, 1997). En el fondo descansa un presupuesto normativo que propone que, dado que los recursos necesarios para cumplir objetivos gubernamentales y no-gubernamentales se encuentran ampliamente distribuidos en diversos sectores sociales, la cooperación es un requisito para enfrentar los problemas de las ciudades. En otras palabras, dado que somos interdependientes, somos corresponsables (Porras, 2019), la cooperación intersectorial en redes es el resultado de la interdependencia (Rhodes, 1997). Por otro lado, en las TRU el motor explicativo es la relación entre la estructura y la agencia (Stone, 2002); lo que explica que el régimen urbano no es la interdependencia inmediata de los recursos para cumplir un determinado objetivo -aunque la «ley de hierro» del régimen urbano requiere proporcionalidad entre objetivos y recursos movilizados para cumplirlos (Stone 2002)-, sino la relación entre la agenda urbana estratégica como producto de la organización social y las acciones de cada actor individual.

Así, los problemas fundamentales de la gobernanza parecen concentrarse en torno a los procesos para acordar e implementar objetivos comunes (i.e. el co-timoneo), enfatizando la agencia de los actores y su capacidad para moldear el entorno. Esto es básicamente lo que Rhodes (1997: I5) llama «redes inter-organizacionales auto-organizadas». Las TRU, por otro lado, subrayan cómo las instituciones (formales e informales), valores y conductas esperadas de la cultura política de una ciudad fomentan una determinada agenda compartida por actores públicos y privados.

\section{ÉLITES ECONÓMICAS LOCALES Y COALICIONES}

Otro problema fundamental en las TRU es el papel asignado a los negocios locales, lo que apunta al problema de quiénes forman parte de las coaliciones del régimen, cómo se forman y cómo funcionan. Stone (1989: 234) argumenta que, dadas las características de las élites de los negocios y otros grupos de estratos socioeconómicos altos, estos son «atractivos como aliados 
a los funcionarios públicos». En el caso de Atlanta, la atracción de la élite económica se extiende más allá del sector público, incluyendo a actores de la sociedad civil organizada y no organizada. En efecto, John (200I. 4I) propone que «los regímenes no son solo acerca de las políticas sobre el desarrollo económico», abriendo la puerta a regímenes urbanos con agendas híbridas (i.e. económico-sociales, económico-culturales, económico-identitarias y de desarrollo sostenible -al estilo de la Agenda 2030 y los Objetivos de Desarrollo Sostenible, ods-), o a miembros de coaliciones que provienen del sector social o de la Investigación y Desarrollo (I+D) - por ejemplo, Organizaciones de la Sociedad Civil (osC), Organizaciones No Gubernamentales (ONG) e instituciones académicas o de investigación que conforman triples hélices- (Mossberger y Stoker, 200I). Las TRU presuponen la importancia de los negocios por default, aunque siempre ha reconocido que el presupuesto de que estos son centrales para el gobierno de las ciudades siempre y en todo lugar no necesariamente se adecua a la evidencia empírica. Diferentes gobiernos pueden perseguir fines distintos y, en consecuencia, privilegiar diversas agendas de política pública (Jones, 1998).

En el fondo, el argumento propone que es imposible gobernar una ciudad solamente con la «maquinaria formal del gobierno», siendo necesaria una «alianza informal entre el gobierno local y la élite empresarial» [...] que constituye «el medio a través del cual se realizan las decisiones de política pública más importantes» (Stone, 1989: 3). Esta alianza es mutuamente beneficiosa si se da en un entorno de democracia liberal y capitalismo, ya que los gobiernos locales pueden operar gracias al aumento de la producción - piénsese e.g. en los empleos e impuestos generados por las actividades económicas preponderantes en una ciudad. El gobierno local depende de los negocios; por eso es natural que estos sean sus interlocutores o aliados.

La élite económica, por otro lado, posee los recursos para influir en las decisiones de política pública a su favor, a través del cabildeo, las contribuciones económicas -en campaña o fuera de ella, legales o ilegales-, y las conexiones entre las élites económicas y políticas que, en algunas ciudades, son indistinguibles entre sí. Sin embargo, las TRU no postulan un determinismo de la influencia económica sobre las instituciones democráticas, sino más bien presenta el problema como un conjunto de tensiones que pueden 
tener diversas soluciones. Si fuera de otra manera, los actores económicos rara vez perderían una «batalla política» (Jones I998: 79-80).

Una crítica inmediata a este modelo es su énfasis en los actores locales sobre otros, tanto de otros niveles de gobierno o poderes, como de representantes de intereses económicos regionales, internacionales y globales. La falta de consideración de variables macropolíticas y macroeconómicas que escapan al control de los actores locales es un límite objetivo que debe ser considerado (Guarneros-Meza, 2006). Sin embargo, reconociendo que variables externas al régimen pueden ser una fuente de dinamismo relevante, el presupuesto de Stone es que en un régimen urbano existe el suficiente espacio de maniobra como para que tanto actores políticos como económicos impulsen una agenda estratégica. Lo importante es que, a nivel local, se deben «movilizar los recursos para tomar e implementar decisiones de gobierno» (Stone, I989: 6). Estos deben ser «proporcionales» a la agenda de política pública prioritaria (Stone, 2002: IO).

Una segunda preocupación expresada en relación con el papel de las élites económicas en el régimen tiene que ver con la tensión entre la «gobernanza neoliberal», mencionada arriba, y la gobernanza territorial -o que considera a actores sociales-. Normativamente hablando, las redes territoriales y sociales deberían tener preeminencia sobre las redes público-privadas vinculadas a servicios públicos y a proyectos de infraestructura -entre otros- (Porras, 2019), y esto se debería asegurar a través del control democrático del gobierno (Stone, 1989: 6). El presupuesto es que, dada cierta estructura social, no importa quién sea electo - pues todo gobierno prefiere crear empleos a perderlos y, en consecuencia, buscará aliarse con los capitales locales. De la misma manera, una influencia indebida de las élites económicas en detrimento de los intereses económicos de la ciudad sería castigada electoralmente (Fainstein y Fainstein, 1986), por lo que -en teoría- el régimen urbano es controlado de manera indirecta por las instituciones democráticas, tanto formales como informales.

Sin embargo, este principio «no es prístino» (Stone, 1989: 6) (i.e. impoluto, inmaculado), en el sentido de que existen mutuas dependencias entre los intereses de los actores gubernamentales y los del sector privado, que hacen difícil que el interés de la ciudad prevalezca en cada una de sus interacciones. La solución, de acuerdo con la literatura del anclaje democrático, es 
asegurar el control de la coalición por parte de funcionarios públicos electos democráticamente; fomentar que los actores privados sean representativos de los negocios más importantes de la ciudad, que la rendición de cuentas siga una lógica territorial y que las interacciones sean lo más democráticas que sea posible (Sørensen y Torfing, 20I4). Esto supone la capacidad de ajustar las coaliciones de manera externa a ellas, lo que no es posible en todas las circunstancias.

\section{AGENDA URBANA IRRESISTIBLE/CONVINCENTE}

Un tercer problema central de las TRU es el del «propósito» del régimen: la agenda público-privada prioritaria para la ciudad. Como bien dice Stone (1989), esta agenda no implica que tanto los gobernantes como los gobernados tengan los mismos objetivos, sino que, dada la experiencia usual de percibir la "condición urbana» como distintos problemas aislados y sin conexión entre sí, el régimen urbano ofrece una visión más integrada sobre ellos (Stone, 2002: 3). De esta manera, el gobierno debe hacer «más que simplemente ofrecer servicios de rutina»; debe «ser capaz de movilizar tanto actores públicos como privados» con base en arreglos informales que, no obstante, son vitales para conducir la ciudad (Stone, 1989: 219). En el centro de la coalición se encuentran temas o problemas que se conciben como medulares. Como dice Stone (2002: 7), en algunas coaliciones unos

[...] socios pueden tener más «cartas» que otros; de ahí que puede no haber equidad. Sin embargo, una agenda irresistible [convincente, compelling] puede consolidar la coalición. Al ser una mezcla de actores gubernamentales y no-gubernamentales, la coalición gobernante no es un grupo que siempre piensa lo mismo. En cualquier coalición existen tensiones a pesar de que sean leales a la alianza y la consideren como un medio para lograr objetivos de política pública importantes [...] Los miembros de la coalición no necesariamente tienen una misma ideología, y sus identidades políticas pueden diferir en dimensiones importantes. Es entonces una agenda compartida la que mantiene la coalición gobernante funcionando. Si la agenda es demasiado estrecha, entonces carecerá de la 
capacidad para establecer una dirección y tendrá una débil pretensión de prioridad [...] Si la agenda es demasiado amplia, puede perder su centro y permanencia. Para que una coalición de gobierno se mantenga, debe poseer credibilidad como un rumbo de acción que puede dar resultados.

$\mathrm{El}$ «conjunto estructurado de relaciones» que existen en la coalición no se da de manera automática (Stone, 1989: 8), sino es algo que se debe construir (Mossberger y Stoker, 200I). Dependiendo de sus características, un régimen urbano puede ser de desarrollo, progresista, radical, conservador o de algún otro tipo, pero su atracción «irresistible» o «convincente» proviene no solo de los contenidos y procesos de la agenda estratégica (la visión que se tiene para la ciudad en términos de objetivos de cogobierno y política pública), sino también de la manera en que esta se define, presenta y reproduce por los actores miembros del régimen y la cultura política de la ciudad. En este sentido, la atracción proviene de un cierto equilibrio que posibilita concebir la agenda como verosímil y factible: un equilibrio entre intereses propios/ comunes, amplitud/estrechez de la agenda, homogeneidad/heterogeneidad política que la hacen «creíble», según lo mencionado arriba. Esto sugiere, como argumenta Bassols (2006a: 36), que lo más sensato es no asumir que existen regímenes urbanos en todas las ciudades, sino que se trata de una excepción más que de una regla.

\section{MEDICIÓN Y RÉGIMEN URBANO}

Por último, tratando de ir más allá del problema de la replicabilidad del régimen urbano y la aproximación del interruptor de luz-mencionada arriba $^{-}$, es evidente que las TRU son útiles no solamente porque explican el crecimiento de Atlanta durante cuatro décadas, sino también porque proponen un modelo que ayuda a entender otras ciudades de Estados Unidos y el mundo. Trascendiendo el contexto concreto que la generó, las TRU -por otro lado- no aspiran a explicar todas las dinámicas urbanas en todas las ciudades: explica más que un caso concreto, pero no todos. El lector perdonará el uso de un término que proviene de la epidemiología, pero el problema de fondo no es uno de prevalencia -i.e. de la proporción de ciudades que 
poseen atributos similares a los de Atlanta, respecto del total de ciudades- ${ }^{-}{ }^{2}$ ni tampoco uno de "peso» o capacidad de generalización -como si una teoría fuera más valiosa entre más casos concretos analizara exitosamente-, sino si las TRU señalan una realidad susceptible de ser cuantificable, es decir, si el régimen urbano puede ser tratado como un conjunto de atributos que se verifican en diversos grados, acercándose o alejándose de ciertos tipos ideales.

Cuando Stone (I989: I8I) usa el término «régimen urbano» lo hace pensando que es algo más que una categoría que ayudará a realizar análisis que visibilicen actores y relaciones que, de otra manera, sería difícil detectar. El régimen urbano no es una perspectiva teórica o un criterio heurístico que existe solamente en la cabeza del investigador, por así decirlo, sino «una realidad concreta», un "comportamiento de actores con una intención explícita». Suponiendo que esto fuera así y que se quisiera evitar el enfoque del interruptor de luz, presuponiendo la utilidad analítica de las TRU, eventualmente se tendrían que abordar los problemas relacionados con la operacionalización de las variables de los regímenes urbanos, la selección de indicadores y la construcción de índices. El desarrollo de herramientas cuantitativas es parte de la frontera del conocimiento que ofrece retos teóricos y metodológicos a las TRU. Esta agenda de investigación está todavía por abordarse de manera sistemática y sustantiva. Sin embargo, la identificación de las variables relevantes realizada por Stone (1989), Mossberger y Stoker (200I), Vélez Dávila (2013) y John (200I) son un buen inicio.

\footnotetext{
${ }^{2}$ Aunque si se quisiera abordar el problema de la prevalencia, John (2001: 50-52) ofrece una serie de prerrequisitos, o condiciones previas de las ciudades, para generar y mantener regímenes urbanos. Para este autor, es más probable que operen regímenes en ciudades donde (a) existe una dimensión local de los intereses económicos; (b) los negocios están relativamente integrados, y bien representados, por actores(as) con cierta visibilidad; (c) se trata de una zona metropolitana (dado que en una ciudad grande y compleja existen más recursos e intereses que pueden ser movilizados en torno a una agenda común prioritaria); (d) existe «una tradición de pragmatismo y confianza en la política local [...] como parte de la cultura política»; y (e) la ciudad analizada es diferente del promedio -aunque sea solo marginalmente-, ya que el sentimiento y las políticas de excepcionalismo pueden facilitar la convergencia sobre una agenda y la generación de coaliciones público-privadas. Este último punto es interesante, ya que sugiere la importancia de factores identitarios que fomentan la unidad/cooperación entre actores públicos y privados.
} 
Por último, los problemas de la medición, tanto en las literaturas de la gobernanza como en las de las TRU, apuntan hacia cómo se concibe la agenda pública de las ciudades, incluyendo su topología (i.e. la estructura básica de interrelaciones de actores, problemas públicos, recursos y narrativas) y, en consecuencia, las mutuas dependencias entre elementos cuantitativos y cualitativos necesarios para entenderla. Plantear las complejidades de medir no implica necesariamente suponer que todo debe medirse, o que todo puede medirse. Sin embargo, considerarlas nos permite identificar a qué se debe dar seguimiento de manera cuantitativa y, en consecuencia, cuáles son las prioridades gubernamentales, sociales y privadas en la agenda urbana. Re-pensar la medición de la gobernanza urbana y de los regímenes urbanos nos obliga a preguntarnos qué debemos medir, para qué queremos medir y cuáles son los usos políticos o de confirmación o cuestionamiento del statu quo que acompañan los esfuerzos de medición (Porras, 2020).

Las TRU ofrecen las categorías/correlaciones de «régimen urbano», "coalición» entre actores públicos y privados, «agenda estratégica» y «ley de hierro" para ayudar a explicar por qué determinadas ciudades crecen/se gobiernan de la manera en que lo hacen. Algunos de los problemas implícitos en ellas están relacionados con la gobernanza, el motor explicativo del régimen urbano (i.e. la relación estructura-agencia), el papel de las élites locales de los negocios, el carácter irresistible/convincente de la agenda y las dimensiones cuantitativas del régimen (i.e. la pregunta de si estos elementos pueden considerarse un continuum con diversos grados de desarrollo). Con estos elementos, las TRU permiten generar análisis y narrativas con cierta utilidad en diversos contextos, independientemente de que se pueda probar incontrovertiblemente la existencia de un régimen urbano operativo.

Este último punto es importante para avanzar en el tratamiento analítico de las TRU. En efecto, la pregunta no debería ser si el hecho social que llamamos «régimen urbano», así como las TRU mismas, pueden viajar bien en el espacio y en el tiempo, como plantea Pierre (2014), sino si las teorías, métodos y evidencia empírica que se desarrollan en torno a los regímenes urbanos nos ayudan a entender (a) cómo se establecen las alianzas público-privadas 
de largo aliento y cómo cambian (i.e. ¡es un asunto de interdependencias o de relación agencia-estructura?); (b) cómo se cogobierna las ciudades con base en alianzas con actores privados o coaliciones más amplias con otros actores; (c) cómo se construyen agendas urbanas que son «irresistibles» o lo suficientemente convincentes como para establecerse como una guía para la acción de muchos actores relevantes, y (d) cómo se pueden conceptualizar diferentes dimensiones del régimen urbano en un continuum gradual que reconozca, por un lado, que su tipo ideal es probablemente inverificable, pero, por el otro, que incluso sus herramientas limitadas y parciales son de utilidad para explicar el gobierno de las ciudades, aunque no se trate de Atlanta, Georgia.

\section{COMENTARIOS FINALES: UNA PEQUEÑA AGENDA DE INVESTIGACIÓN}

Una aproximación analítica a las TRU permite escapar a las trampas del enfoque del interruptor de luz, que pone énfasis en la verificación en campo de un modelo preestablecido para luego simplemente aplicar implicaciones teóricas, empíricas y metodológicas. Una aproximación analítica va más allá de las expectativas normativas de los modelos, reconociendo que las categorías y correlaciones propuestas tienen una utilidad parcial, explicando algunas dimensiones y procesos del cogobierno público-privado de las ciudades.

Las TRU son útiles para entender a las ciudades si se consideran de manera amplia, como un conjunto de categorías, enfoques y correlaciones que enfatizan cómo las autoridades locales necesitan de alianzas con actores privados para obtener los objetivos de la agenda estratégica de la ciudad. El mapeo específico de los regímenes requiere del análisis de actores; la realización de entrevistas e investigación documental para definir las fronteras territoriales, escalares y de contenidos de la agenda; su duración -cómo y en qué medida se ha mantenido más allá de las administraciones locales-, los componentes sociales de las alianzas, los liderazgos personales e institucionales y un catálogo de las asociaciones público-privadas actualmente funcionando, tanto formales como informales (Bassols, 2006. 9). Adicionalmente, es necesario 
realizar investigación en torno a los problemas fundamentales mencionados. Una pequeña agenda de investigación podría incluir lo siguiente:

Necesitamos continuar con el trabajo de investigación de campo tratando de analizar cómo la cultura política, compartida entre actores públicos y privados, da por resultado arreglos informales concretos que impulsan agendas específicas. Según las TRU, las relaciones/tensiones entre estructura y agencia pueden explicar por qué la reproducción del sistema social genera tales coaliciones. Esto, sin embargo, debe explorarse con mayor profundidad, usando herramientas analíticas de cultura cívica; es decir, es necesario bajar a nivel de «tuercas y tornillos» para entender cómo funciona el motor que genera, mantiene y cambia los regímenes urbanos.

El posible conocimiento obtenido tendrá utilidad, independientemente de si se puede argumentar o no la presencia de un régimen urbano en un contexto determinado, particularmente porque le dará herramientas a las TRU para dialogar con otros motores explicativos -más prominentemente con la interdependencia, en el caso de las literaturas de la gobernanza. Como se puede apreciar, el problema de fondo es evaluar la potencia explicativa de la perspectiva de las ciencias políticas (basada en la racionalidad de la cooperación público-privada que resulta de la interdependencia) frente a la perspectiva sociológica (basada en las relaciones entre la agencia y la estructura: entre las decisiones de los actores y la cultura política que impulsa una determinada agenda «irresistible»).

Otro punto importante de la agenda de investigación es el mapeo/confirmación de los actores relevantes en las coaliciones (élites económicas locales). Dada la naturaleza informal del régimen, las entrevistas semiestructuradas o focus groups a realizar, así como otra investigación de tipo documental, tendrían que evidenciar el intercambio/movilización de los recursos necesarios para mantener la agenda urbana estratégica. Esto probablemente implicaría demostrar que el intercambio de información, recursos financieros, instrumentos legales, expertise técnico y otros bienes públicos -como la confianza interpersonal e institucional- entre ciertos actores públicos y privados se realiza con el objetivo de hacer avanzar la agenda urbana estratégica. Un reto teórico-metodológico es cómo interpretar las TRU a la luz de actores que no son locales, pero que, sin embargo, tienen una gran relevancia local. 
En tercer lugar, los procesos de definición y mantenimiento de las agendas urbanas estratégicas deben ser entendidos de mejor manera. Como se ha mencionado, Stone (1989) no propone un determinismo en la reproducción del sistema social y la agenda urbana resultante. El ámbito de la agenda es uno de agencia y, en consecuencia, de libertad de los actores para alcanzar acuerdos e incluso ir en contra de lo que es aceptado por la mayoría. En consecuencia, la agenda urbana estratégica/prioritaria debe interpretarse como una actividad política, la cual «provee la oportunidad para que la gente actúe conjuntamente para cambiar [...] el curso de los eventos» (Stone, 2002: I2). Los procesos políticos que colocan ciertos problemas en la agenda pública deben analizarse a la luz de su capacidad para generar entendimientos comunes de estos, a la luz de las recomendaciones de Alford y Head (2017) para lidiar con problemas complejos. La acentuación de los problemas urbanos y metropolitanos en torno al crecimiento, los servicios públicos, la competitividad, la atracción de inversiones, el desarrollo sostenible y el cambio climático, sugieren un cierto potencial de la Agenda 2030 para promover agendas amplias, que incluyan un gran número de actores.

La ciudad dual - presente en todas nuestras ciudades-cuestiona la calidad de una agenda urbana estratégica que termina por empoderar a actores que no son necesariamente locales, ni mayoritarios. Como dice Stone (2002: 13),

si las participaciones en la gobernanza son tan desiguales que algunos mantienen un lugar especial de privilegio mientras que otros no reciben ninguna consideración, la política reprueba el examen elemental de la democracia. Las agendas estratégicas de las localidades son un examen. Si son débiles o no existen, la ciudadanía carece de poder político y un aspecto importante de la democracia no está trabajando [...] Aún más, si las agendas locales sirven solamente a los intereses inmediatos de los negocios y no atienden los asuntos de la exclusión social, es la parcialidad [bias], no la democracia, la que trabaja.

En este sentido, la agenda urbana estratégica puede fracasar al no incorporar de manera efectiva criterios y actores territoriales y sociales que sean capaces de matizar y filtrar las demandas del mercado. 
Finalmente, es necesario hacer mayor investigación en el desarrollo de instrumentos de medición de las dinámicas formales e informales de las coaliciones. Esto podría incluir la operacionalización y selección de indicadores de diferentes variables, tales como

a) La composición de las coaliciones del régimen, diferenciando por tipo de actor y -en el caso del uso de grafos- la ponderación de la clase de recursos intercambiados, así como su importancia (cantidad).

b) El mapeo de los elementos que componen la agenda urbana estratégica, en términos de políticas públicas y privadas (e.g. la inversión en desarrollo de infraestructura, los diferentes programas implementados, el número, tipo y calidad de las alianzas público-privadas, los resultados en el posicionamiento de las ciudades y la atracción de inversiones, entre otros).

c) Intentar alguna medida de la ley de hierro, tratando de vincular los recursos movilizados con la agenda prioritaria y-crucialmente- con los valores existentes en la cultura cívica del lugar. Esto ayudaría a explorar algunos problemas que en la actualidad permanecen claramente en el ámbito de lo contrafactual, pero que pudieran ser determinantes para mejorar la calidad de vida de las ciudades. Por ejemplo, si los habitantes de la ciudad apoyaran irresistiblemente políticas de desarrollo sostenible, ¿sería posible cambiar el tipo de agenda estratégica para la ciudad? ¿Se podría desarrollar un verdadero régimen urbano en torno a esta? ¿Es sensato pensar en realizar tal cambio, dada la historia de nuestras ciudades?

Evidentemente, estas son preguntas con muy diverso grado de importancia y factibilidad. Sin embargo, son relevantes, en la medida en que son resultado de la consideración de las ciudades a la luz de las TRU. Responder algunas de ellas podría ser una contribución sustancial al entendimiento del gobierno de las ciudades, pero también del régimen urbano y su utilidad teórica, empírica y metodológica. 


\section{REFERENCIAS}

Aguilar, L.F. (2006). Gobernanza y gestión pública. Ciudad de México: Fondo de Cultura Económica.

Aguilar, L.F. (2019). La gobernanza del sector público. Zapopan: El Colegio de Jalisco.

Alford, J. y Head, B.W. (20I7). Wicked and less Wicked Problems: a Typology and a Contingency Framework. Policy and Society, 36(3), 397-4I3.

Ansell, C. y Torfing, J. (20I6). Epilogue: The Current Status and Future Development of Governance Theories. En C. Ansell y J. Torfing (eds.). Handbook on Theories of Governance. Cheltenham (RU): Edward Elgar Publishing Limited, 55I-559.

Bassols, M. (2006). Prefacio. En M. Bassols (ed.). Explorando el régimen urbano en México: un análisis metropolitano. Tijuana, Ciudad de México: El Colegio de la Frontera Norte, Universidad Autónoma Metropolitana, Unidad Iztapalapa, 7-II.

Bassols, M. (2006a). Los entresijos del poder urbano. En M. Bassols (ed.). Explorando el régimen urbano en México: Un análisis metropolitano. Tijuana, Ciudad de México: El Colegio de la Frontera Norte, Universidad Autónoma Metropolitana, Unidad Iztapalapa, 27-59.

Cadena Inostroza, C. y Salgado Locela, L.H. (2017). Redes y capacidades de actores en torno a comités independientes de agua potable: el caso de San Felipe Tlalmimilolpan, Toluca, México. Revista de El Colegio de San Luis. I3, 62-90.

Ciccolella, P. y Mignaqui, I. (20I6). Metrópolis latinoamericanas: fragilidad del Estado, proyecto hegemónico y demandas ciudadanas. Algunas reflexiones a partir del caso de Buenos Aires. En A. Iracheta (ed.). Metrópolis y gobernanza: bases conceptuales y experiencias. Ciudad de México: Siglo XXI, Anthropos, I7I-I95.

Davies, J. (20II). Challenging Governance Theory: from Networks to Hegemony. Bristol: The Policy Press.

Davies, J. y Blanco, I. (20I7). Austerity Urbanism: Patterns of Neo-liberalisation and Resistance in Six Cities of Spain and the uk. 
Environment and Planning A. o. <https://journals.sagepub.com/ doi/pdf/Io.II77/0308518XI7701729>.

Emerson, K. y Nabatchi, T. (2015). Collaborative Governance Regimes. Washington D.C.: Georgetown University Press.

Fainstein, N.I. y Fainstein, S.S. (1986). Regime Strategies, Communal Resistance, and Economic Forces. En S. S. Fainstein, N. I. Fainstein, R. C. Hill, D. Judd, y M. P. Smith (eds.). Restructuring the City: The Political Economy of Urban Redevelopment. Nueva York: Longman, 245-282.

Gash, A. (2016). Collaborative Governance. En C. Ansell y J. Torfing (eds.). Handbook on Theories of Governance. Cheltenham (RU): Edward Elgar Publishing Limited, 454-467.

Guarneros-Meza, V. (2006). Los pros y los contras desde una perspectiva europea. En M. Bassols (ed.). Explorando el régimen urbano en México: un análisis metropolitano. Ciudad de México, Tijuana: El Colegio de la Frontera Norte, Universidad Autónoma Metropolitana Unidad Iztapalapa, Plaza y Valdés, 6I-84.

Guarneros-Meza, V. (marzo, 2007). Urban Governance and Participation in Central Mexico. Development. 50(I), I04-I09.

Guarneros-Meza, V. (2008). Local Governance in Mexico. The Case of Two Historic-Centre Partnerships. Urban Studies. 5(5-6), IoIIIO35.

Guarneros-Meza, V. (20II). Localismo, neoliberalismo y poder. Élites urbanas y prácticas culturales en Polonia y México. En M. Bassols y C. Mendoza (eds.). Gobernanza. Teoría y prácticas colectivas. Ciudad de México: uam Iztapalapa, Anthropos, I77-207.

John, P. (200I). Local Governance in Western Europe. Londres: Sage.

Jones, B.D. (1998). Bureaucrats and Urban Politics: Who Controls? Who Benefits? En D. Judge, G. Stoker y H. Wolman (eds.), Theories of Urban Politics. Londres: Sage, 72-95.

Judge, D., Stoker, G. y Wolman, H. (1998). Urban Politics and Theory: an Introduction. En D. Judge, G. Stoker y H. Wolman (eds.). Theories of Urban Politics. Londres: Sage, I-I2.

Kooiman, J. (2003). Governing as Governance. Londres: Sage. 
McCann, E. (20I7). Governing Urbanism: Urban Governance Studies I.O, 2.0 and beyond. Urban Studies. 54(2), 312-326.

Mossberger, K. y Stoker, G. (julio 200I). The Evolution of Urban Regime Theory: The Challenge of Conceptualization. Urban Affairs Review, 36(6), 810-835.

Palumbo, A. (2015). Situating Governance. Context, Content, Critique. European Consortium for Political Research. Colchester: Hythe Quay.

Pierre, J. (20II). The Politics of Urban Governance. Nueva York: Palgrave Macmillan.

Pierre, J. (20I4). Can Urban Regimes travel in Time and Space? Urban Regime Theory, Urban Governance Theory, and Comparative Urban Politics. Urban Affairs Review. 5o(6), 864-889.

Porras, F. (2019). Gobernanza: propuestas, limites y perspectivas ( $2^{\mathrm{a}}$ ed.). Ciudad de México: Instituto Mora, Consejo Nacional de Ciencia y Tecnología.

Porras, F. (2020). Gobernanza y medición: algunos problemas. En C. A. Navarrete, A. A. Guevara y J. A. Demerutis (eds.). Nuevas formas de acción pública metropolitana. Narrativas y modelos. Zapopan, Jalisco: El Colegio de Jalisco, Red Gobernanza Metropolitana, 45-80.

Rhodes, R.A.W. (1997). Understanding Governance. Policy Networks, Governance, Reflexivity and Accountability. Maidenhead, Berkshire: Open University Press.

Rhodes, R.A.W. (2000). Governance in Public Administration. En Pierre, J. (ed.). Debating Governance. Authority, Steering and Democracy. Oxford: Oxford University Press, 54-90.

Skelcher, C., Klijn, E.-H., Kübler, D., Sørensen, E. and Sullivan, H. (20II). Explaining the Democratic Anchorage of Governance Networks. Administrative Theory and Praxis, 33(I), 7-38.

Sørensen, E. y Torfing, J. (20I4). Assesing the Democratic Anchorage of Governance Networks. En S. Griggs, A. J. Norval y H. Wanegaar (eds.). Practices of Freedom: Decentred Governance, Conflict and Democratic Participation. Cambridge: Cambridge University Press, I08-I36. 
Stoker, G. (1998). Regime Theory and Urban Politics. En D. Judge, G. Stoker y H. Wolman (eds.). Theories of Urban Politics. Londres: Sage, 54-7I.

Stone, C.N. (1989). Regime Politics. Governing Atlanta, 1946-1988. Lawrence, Kansas: University Press of Kansas.

Stone, C.N. (1998). Political Leadership in Urban Politics. In D. Judge, G. Stoker y H. Wolman (eds.) Theories of Urban Politics. Londres: Sage, 96-II6.

(Marzo, 2002). Urban Regimes and Problems of Local Democracy. Ponencia presentada en el Workshop 6, Institutional Innovations in Local Democracy. Conferencia llevada a cabo en ECPR Joint Sessions, Turín, Italia.

Vélez Dávila, M.I.G. (20I3). Poder local en San Luis Potosí, I980-2009. Una aproximación a través del régimen urbano (Tesis doctoral). El Colegio de San Luis, A. C., San Luis Potosí, S.L.P.). 